\title{
Automatic Placement of Outer Volume Suppression Slices in MR Spectroscopic Imaging of the Human Brain
}

Number of words: 5335

${ }^{1}$ Manel Martínez-Ramón, ${ }^{1}$ Ascensión Gallardo-Antolín, ${ }^{1}$ Jesús Cid-Sueiro, ${ }^{2}$ Gregory

L. Heileman, ${ }^{3}$ Kaung-Ti Yung, ${ }^{3}$ Weili Zheng, ${ }^{3}$ Chenguang Zhao, ${ }^{3,4}$ Stefan Posse

1: Dept. of Signal Processing and Communications, Universidad Carlos III de Madrid, Leganés, Madrid, Spain.

2: Dept. of Electrical and Computer Engineering, The University of New Mexico, Albuquerque, NM, USA.

3: Dept. of Neurology, The University of New Mexico School of Medicine, Albuquerque, NM, USA.

4: Dept. of Physics and Astronomy, The University of New Mexico, Albuquerque, NM, USA.

Corresponding author: Manel Martínez-Ramón, Dept. of Signal Processing and Communications, Universidad Carlos III de Madrid, Avda Universidad, 30, 28911 Leganés-Madrid, Spain. E-mail address: manel@ieee.org.

Running Title: Automatic placement of OVS slices in MRSI.

March 25, 2009 


\begin{abstract}
Spatial suppression of peripheral regions (outer volume suppression) is used in MR spectroscopic imaging (MRSI) to reduce contamination from strong lipid and water signals. The manual placement of outer volume suppression slices requires significant operator interaction, which is time consuming and introduces variability in volume coverage. Placing a large number of outer volume saturation bands for volumetric MRSI studies is particularly challenging and time consuming, and becomes unmanageable as the number of suppression bands increase. In this study a method is presented that automatically segments a high-resolution MR image in order to identify the peripheral lipid containing regions. This method computes an optimized placement of suppression bands in three dimensions, and is based on the maximization of a criterion function. This criterion function maximizes coverage of peripheral lipid containing areas and minimizes suppression of cortical brain regions, and regions outside of the head. Computer simulation demonstrates automatic placement of sixtenn suppression slices to form a convex hull that covers peripheral lipid containing regions above the base of the brain. In vivo metabolite mapping obtained with short TE proton-echo-planar spectroscopic-imaging (PEPSI) shows that the automatic method yields a placement of suppression slices that is very similar to that of a skilled human operator in terms of lipid suppression and usable brain voxels.
\end{abstract}

Key words: MR, spectroscopic imaging, outer volume suppression, image segmentation, automation, morphological processing. 


\section{Introduction}

In proton MR spectroscopic imaging (MRSI) of the brain, it is necessary to suppress overwhelming lipid and water signals from peripheral regions around the brain in order to prevent spectral contamination in the volume of interest when computing the point spread function.

Prelocalization of a rectangular box inside the brain using point resolved spectroscopy (PRESS) or stimulated echo acquisition mode (STEAM) volume selection is still widely used, but the loss in volume coverage is undesirable and the selection of large volumes at high magnetic field suffers from considerable chemical shift displacement artifact at the edges of the PRESS and STEAM box. Due to its ease of use, lipid nulling using the Short T1 Inversion Recovery (STIR) method (1-4) is frequently used for whole brain MRSI (5).. However, this leads to a sensitivity loss in all metabolites, and the efficiency of lipid suppression is limited due to the T1 dispersion between lipid resonances. This method is thus most suitable for intermediate and long echo time (TE) acquisition, which further reduces sensitivity at high field as a result of decreases in $T_{2}$ with increasing field strength (6). Frequency selective suppression has been proposed as an alternative and implementations using echo dephasing, such as MEGA or BASING $(7,8)$, have shown powerful suppression performance. However, a disadvantage of these techniques is the substantial elongation of TE, suppression of lactate and macromolecular signal originating from brain tissue and sensitivity to B0- and B1-inhomogeneity.

Spatial presaturation of the lipid containing peripheral regions (9) allows the use of short echo time, which is desirable at high fields to maintain sensitivity as the T2 values of metabolites decrease with field strength. A major limitation of spatial lipid suppression for practical applications is the precise (manual) placement of a large number of presaturation slices to cover peripheral regions while minimizing loss of cortical signals.

A human operator can place eight suppression bands around a two dimensional slice (orthin slab) of the brain and slight tilts of these suppression slices in the third dimension is manageable using orthogonal views. However, placing outer volume saturation (sat) bands across large peripheral areas for volumetric MRSI studies is challenging and time consuming. Thus, manual placement becomes unmanageable as the number of suppression bands increase.

Optimization of the suppression band placement also has to take into account constraints, such as minimization of the suppression slice thickness to minimize chemical shift artifacts and the transition width of the suppression slice profile. Therefore, finding an optimal solution using manual placement is very challenging and performance varies according to the operator. 
There is currently no automated method available to position suppression slices for brain MRSI, though a related method $(10,11)$ to select an irregularly shaped volume inside the brain using multiple sat bands with a block optimization algorithm has been presented. In (10), the authors use PCA and fast standard 3D (is a word missing here?) to place sat bands around masses. Their work is intended to be applied to measurements in and around brain tumors, breast lesions and prostate cancer, not to the measurement of whole organs.

In this paper we present an automatic method for placing up to 16 outer volume suppression slices for brain MRSI. The slices form a convex hull that covers the peripheral lipid containing regions above the base of the brain. The method dramatically reduces human operator interaction and utilizes a mathematical algorithm that finds an optimal solution by iteratively optimizing a cost function. The novelty of this algorithm is that it automatically maximizes the coverage of the peripheral lipid containing regions, while at the same time minimizing loss of brain tissue due to suppression, and simultaneously limiting the coverage of volumes outside of the head to minimize artifacts in the transition region of the suppression slices. This method takes into account the fact that lipid suppression in the transition band is incomplete, and that partial suppression of metabolite signals in the transition band impairs spectral quantification. Since iterative optimization may produce different solutions depending upon the initial positions of the saturation bands and the shape of the head, we developed a procedure to find positions that produce reproducible results after a few iterations of the algorithm. We describe in vivo 3D metabolite mapping with short TE proton-echo-planar-spectroscopic-imaging (PEPSI) and quantitatively compare the performance of suppression slice placement by a human operator to that of our automated placement method.

\section{Methods}

\section{Theory}

Lipid suppression slices are described by a direction vector $\mathbf{w}_{\mathrm{i}}$ that is orthogonal to each slice, a thickness $d_{i}$, and a distance to the origin of the magnet. These slices, covering the periphery of the brain, form a closed helmet that can be modeled as an inner and an outer hull. The intersection of the helmet with the gray matter and lipid regions is computed using a lipid mask, which consists of a 3D image in which voxels are set to 1 if they belong to lipid tissue, and 0 otherwise. The parameters of the hulls (Figure 1) are iteratively modified in order to maximize coverage of the peripheral lipid containing areas and minimize suppression of cortical brain regions and of regions outside of the head. The procedure consists of the following steps:

1. Identification of the voxels that are likely to be part of peripheral lipid regions. 
2. Identification of the inferior edge of the MRSI volume in brain tissue that excludes nonbrain regions in the MRSI volume. This inferior edge enables placement of an inferior suppression slice to remove possible spatial contamination from inferior regions.

3. Fast (coarse) adjustment of up to 15 suppression slices placed in a convex hull around the upper cerebrum to obtain initialization parameters for the subsequent optimization step.

4. Fine adjustment of the suppression slices using a gradient descent over a cost function.

5. An optional manual adjustment step of the slice parameters to accommodate further constraints, such as avoiding areas with excessive magnetic field inhomogeneity.

The current implementation supports up to sixteen suppression slices, which is consistent with the hardware constraints (RF power, SAR and gradient strength) of current MRSI technology at 3T. Further, the method is currently targeted at the upper cerebrum, which exhibits acceptable magnetic field homogeneity. However, there is no intrinsic limitation of the methodology for extending coverage to major parts of the brain using a convex hull that additionally covers the back side of the cerebellum. Mid-brain and frontal structures are more difficult to suppress using planar slices and spatially tailored suppression methods for these regions are under investigation.

\section{Step 1: Identification of peripheral lipid regions}

Segmentation software tools, such as Statistical Parametric Mapping (SPM) $(12,13)$ or fMRIB Software Library (FSL) (14) can be used to obtain images of gray matter, white matter and CSF based on high-resolution MRI, which are typically $\mathrm{T}_{1}$-weighted MRI scans. Subtracting these tissue masks from the original MRI results in an image of the periphery. An intensity threshold converts the difference image into a lipid mask. However, the mask may occasionally be corrupted by small artifacts due to image intensity inhomogeneity, corresponding to voxels belonging to the brain, or phase encoding artifacts outside of the brain that are wrongly labeled as lipid. These artifacts may degrade the performance of the slice positioning algorithm, leading to excessive suppression of brain tissue or overly broad suppression slices with large transition regions with partly suppressed lipids. Spurious voxels are removed by requiring that all lipid voxels be connected to form a single contiguous suppression region in three dimensions.

Spurious voxel removal is accomplished through the use of well-known morphological operations (15). Lipid voxels are identified by selecting the largest connected component in the lipid mask, and removing all other voxels. In this process, a six-connectivity operator was used: Specifically, let the neighbors of voxel $\mathrm{x}=(\mathrm{x} 0, \mathrm{x} 1, \mathrm{x} 2)$ be $(\mathrm{x} 0 \pm 1, \mathrm{x} 1, \mathrm{x} 2),(\mathrm{x} 0, \mathrm{x} 1 \pm 1, \mathrm{x} 2)$ and $(\mathrm{x} 0, \mathrm{x} 1$ , $\mathrm{X} 2 \pm 1$ ). Nonzero voxels $\mathrm{Xa}$ and $\mathrm{xb}$ belong to the same connected component only if there exists a path from $\mathrm{xa}$ to $\mathrm{xb}$ that travels across neighboring nonzero voxels. 
In order to select the largest connected component, all connected components are identified using the following general procedure:

1. Scan all lipid mask voxels, assigning preliminary labels to nonzero voxels, and

2. Relabel voxels using the union-find algorithm (16), which iteratively scans all image voxels, finds the components connected to each voxel, and merges regions (by relabeling) if two voxels with different labels are neighbors (according to the "sixconnectivity" rule (14)).

\section{Step 2: Identification of the inferior edge of the MRSI volume and placement of the inferior suppression slice}

The peripheral lipid region has two parts with different geometrical properties: (1) the upper part is nearly convex and will be covered with up to fifteen suppression slices, and (2) the lower part is non-convex and exhibits a complex geometrical structure, with air-filled spaces which must be covered with a single suppression slice. The aim is to identify the regions as either belonging to or not belonging to the non-convex part of non-brain tissue inferior to the brain with a geometrical analysis of the interface between the brain and the lipid.

Inferior non-brain, non-convex regions are connected to peripheral lipid regions through paths in inferior image regions. Thus, if the lipid mask is displayed in an axial slice that moves from the top of the head towards the foot, disconnected "lipid" regions emerge below the brain which start merging with the peripheral lipid regions as the slice moves further inferior. The algorithm processes each axial slice position by identifying the voxels from the peripheral lipid regions (i.e., the convex part of the hull). The remaining voxels are assigned to the non-convex volume. If a non-convex area merges with the lipid section at a given axial slice location, the non-convex volume is extended towards the bottom. This process is illustrated in Figure 2.

Once the non-convex region is identified, it is covered with the lower suppression slice to close the bottom of the convex hull using a straightforward linear program.

\section{Step 3: Coarse adjustment of the suppression slices}

Starting from the center of the intersection of the peripheral lipid mask with the inferior suppression slice defined in step 2, fifteen rays are traced above the inferior suppression slice to intercept the peripheral lipid mask: eight rays have elevation angles of $90^{\circ}$ and azimuth angles equally spaced from $-180^{\circ}$ to $180^{\circ}$ to define a lower ring of eight planes; six rays have elevation angles of $30^{\circ}$ and azimuth equally spaced from $-180^{\circ}$ to $180^{\circ}$ to form an upper ring of six planes; and a ray with $0^{\circ}$ elevation angle perpendicular to the bottom plane to place the top plane. The intersecting voxels $c_{j}, 1 \leq \mathrm{j} \leq 15$, of all rays with respect to the inner surface of the lipid mask are 
found.

A cone with solid angle $\varphi$ is traced from each of the voxels $c_{j}$, towards the outer surface of the lipid, and the set of voxels of the external surface of the lipid that lie inside each of the cones are used to adjust a regression plane. Each adjusted plane is roughly tangent to the external surface.

Finally an inner plane that contains voxel $c_{j}$, parallel to each one of the previously adjusted planes, is found. Each pair of planes defines a slice.

\section{Step 4: Fine adjustment of the suppression slices}

The above operation defines a helmet that roughly covers the lipid. These hulls must be adjusted in order to obtain maximum coverage of peripheral lipids while minimizing coverage of brain tissue and regions outside of the head (hereafter referred to as the "non-lipid regions"). A cost function related to the intersection of the suppression slices with the underlying anatomy is applied to update the slice parameters. The goal is to minimize the intersection of the volume of the helmet with the gray matter $\left(U_{b}\right)$ and with the volume outside of the head $\left(U_{o}\right)$, and to maximize the intersection of the helmet with the peripheral lipid area $\left(U_{l}\right)$. Thus, the cost function is:

$$
L=a U_{b}-b U_{l}+c U_{o}
$$

Coefficients $a, b, c$ provide different weights to the cost associated with the three intersections. Small values for coefficients $a$ and $c$ and a higher value for coefficient $b$ ensures that the uncovered lipid areas are penalized more than suppressed gray matter or suppression slices extending outside of the head.

An iterative computation of the gradient of $L$ with respect of all parameters of the hulls (the parameters $\mathbf{w}_{\mathrm{i}}$ and $\mathrm{d}_{\mathrm{i}}$ of each band) is performed to update the parameters in the opposite direction of the gradient. Since there is no analytical expression for the volume of the peripheral lipid regions, a numerical procedure is employed, with the following steps:

- for $\mathrm{t}=1$ to $\mathrm{T}$

○ for all parameters

- Increase parameter $p_{j}$ a quantity $\delta_{j}$

- Compute $\mathrm{L}\left(p_{j}+\delta_{j}\right)$

- Decrease parameter $p_{j}$ a quantity $\delta_{j}$

- Compute $\mathrm{L}\left(p_{j}-\delta_{j}\right)$ 


$$
\begin{aligned}
& \text { - Compute } \Delta_{j}=\mathrm{L}\left(p_{j}+\delta_{j}\right)-\mathrm{L}\left(p_{j}-\delta_{j}\right) \\
& \text { - Update parameter } p_{j} \text { with the quantity } \mu \Delta_{j} \\
& \circ \text { end }
\end{aligned}
$$

- end

In order to obtain the values of $U_{b}, U_{o}$ and $U_{l}$, the algorithm computes the intersection between the voxels of the lipid mask obtained in step 2 and those voxels that belong to the volume of the helmet. A voxel $\mathrm{x}$ belongs to the suppression volume if it satisfies the following two conditions:

$$
\begin{gathered}
\mathbf{w}_{\mathrm{i}}^{\mathrm{T}} \mathrm{x}+\mathrm{b}_{\mathrm{i}, 1} \geq 0 \text {, for some } \mathrm{i} \\
\mathbf{w}_{\mathrm{i}}^{\mathrm{T}} \mathrm{x}+\mathrm{b}_{\mathrm{i}, 2} \leq 0, \forall \mathrm{i} .
\end{gathered}
$$

where $\mathbf{w}_{\mathrm{i}}, \mathrm{b}_{\mathrm{i}, 1}$ and $\mathrm{b}_{\mathrm{i}, 2}$ are the parameters of each suppression slice (see Figure 1).

The entire process uses a large amount of memory and large number of arithmetic operations, and the initial run time estimations resulted in unacceptable processing times, making the algorithm impractical. In order to reduce the memory usage and the computing time, each set of eight bits was stored in a byte of memory. A library of special operators was then coded to perform the operations required by the method. These improvements reduced the running time by a factor of ten, and the physical memory usage by a factor of eight. Further optimizations reduced the required computation time by a factor of twenty.

\section{Step 5: Optional manual adjustment of the suppression slices}

A final manual adjustment of the suppression slices based on visual inspection of the slice positions was implemented to allow optimization of the coverage of peripheral lipid regions and to allow exclusion of regions with strong magnetic field inhomogeneity. This was found to be useful, as manual adjustments are much faster than running the algorithm until total coverage is obtained.

\section{Method implementation}

DICOM magnetization prepared rapid gradient echo (MP-RAGE) images were converted to NIFTI1 format in RAS orientation and were intensity inhomogeneity corrected using N3 (17). The image of peripheral structures was obtained in FSL by subtracting the brain (extracted using BET) from the inhomogeneity corrected NIFTI images. Saturation slices were automatically placed on the skull image using a custom designed GUI by providing their centers and normal vectors in the images space (voxel). These center and normal vectors 
were transformed to the scanner coordinate system (mm) in PLS orientation using the voxel to RAS transformation information in the MP-RAGE header.

The processing pipeline was implemented in Matlab, except for the fine adjustment procedure, which was coded in $\mathrm{C}++$ and then integrated into the Matlab code. The steps described above were embedded into a graphical user interface (GUI) that allows the user to execute and monitor all steps of the pipeline, graphically and numerically examine the results, and store or retrieve the generated data and parameters. The GUI has been designed using the Matlab GUIDE package.

The methodology was designed and tested offline for sixteen suppression slices. However, for actual placement of suppression slices in MRSI scans only the eight suppression slices from the lower ring were used due to limitations of the pulse sequence software. The initial ray angles of these slices were decreased to $75^{\circ}$ to maximize volume coverage for 3D MRSI in the upper cerebrum.

The PEPSI pulse sequence described in (18) was modified to read the parameters for eight variable suppression slices from the text file generated in the previous step. This enabled direct visualization of the suppression slices superimposed on high-resolution MRI using the scanner graphical user interface.

\section{In vivo experiments}

\section{Subjects and scanners}

Six healthy subjects participated after giving institutionally reviewed informed consent. Data were collected on a 3T TIM Trio scanner (Siemens Medical Solutions, Inc.) equipped with Avanto gradient system and 12 channel circularly polarized head coil.

\section{Data Acquisition}

PEPSI data sets were acquired from a $45 \mathrm{~mm}$ thick para-axial slab in AC/PC orientation encompassing anterior cingulated and supra-ventricular brain, using 1st and 2nd order autoshimming and the following parameters: TR: 2 s, TE: $11 \mathrm{~ms}$, FOV: 226x226x55 mm, spatial matrix: $32 \times 32 \times 8$ pixels, nominal voxel size: $0.3 \mathrm{~cm}^{3}$, scan time with weighted sampling in the Y-Z plane: 4.5 min using single average. In addition to the water-suppressed acquisition (WS), a non-water suppressed (NWS) reference scan with 1 average was collected. High resolution $\mathrm{T}_{1}$-weighted MP-RAGE scans $(\mathrm{TR}=1810 \mathrm{~ms}$, $\mathrm{TI}=900 \mathrm{~ms}, \mathrm{TE}=2.52 \mathrm{~ms}$, flip angle $=8^{\circ}, 176$ sagittal slices, matrix size $\left.256 \times 256\right)$ with isotropic resolution $\left(1 \mathrm{~mm}^{3}\right)$ were acquired for tissue segmentation. Multi-slice $\mathrm{T}_{2}$-weighted Turbo Spin-Echo scans with the 
same slice orientation were acquired for manual placement and for verifying the automatic placement of the saturation bands on the scanner user interface. The setup time for positioning the suppression slices took approximately 15 minutes after the acquisition of the MP-RAGE scan.

The current Siemens software is such that the RF pulse shape is fixed, the RF pulse amplitude is maximized to minimize RF pulse duration and the slice thickness is changed by changing the gradient amplitude. Increasing the slice thickness without decreasing the gradient amplitude or widening the transition band width by just choosing an RF pulse with higher time-bandwidth product is not implemented due to RF power limitations and due to the need to minimize the duration of the suppression slice modules to minimize T1 relaxation effects.

\section{MRSI preprocessing and reconstruction}

Data reconstruction was performed as described previously (18) using separate processing of odd and even echoes. Automatic zero-order phase correction and frequency shift correction based on the water signal in the NWS reference scan were applied on a voxel-by-voxel basis. Odd and even spectra were then summed to obtain NWS and WS spectral arrays. Spectra were converted to time-domain by an inverse Fourier transform and exported for LCModel fitting using an amplitude threshold to exclude voxels with low signal-to-noise ratio originating from regions outside of the brain.

\section{Spectral quantification}

Localized spectra were quantified using LCModel fitting (Version 6.1) (19) and simulated spectral basis sets as described previously (18). Spectra were fitted in the spectral range between 0.2 and $4.2 \mathrm{ppm}$ in reference to the NWS data using "water-scaling". A Cramer-Rao lower bound (CRLB) threshold of $20 \%$ and maximum spectral line width threshold of 0.1 ppm were used.

To compare the performance of automated placement against a human operator, two metrics were used: (a) the integrated residual lipid signal divided by the integrated creatine signal across the entire spectroscopic slice and (b) the number of usable voxels with clearly identifiable metabolite signals. 


\section{Results}

\section{Offline characterization of free parameter robustness and fine adjustment}

An example of coarse adjustment is shown in Figure 3. Robustness of the fine adjustment procedure has been tested with different coarse adjustment parameters that produce several initial positions of the bands. Coarse adjustment has been run with several values for elevation angles of the top ring of planes from $\pi / 6$ to $\pi / 3$. Different cone angles $\varphi$ from $\pi / 6$ to $\pi / 2$ were used. The resulting values of the inner plane distances to the origin were changed by a factor $\beta$, from 0.8 to 1 , in order to improve the lipid coverage of the coarse adjustment. Different values of parameters $a, b$ and $c$ of the cost function as well as the fine adjustment step $\mu$ were tested. Figure 4 shows the evolution of the lipid and non-lipid coverage for different values of $\beta$. The chosen parameters are $a=c=0.2, b=10, \mu=1.25 \cdot 10-4, \beta=0.9, \varphi=\pi / 2$ and $\theta=\pi / 4$. The result of the fine positioning is in Figure 5.

\section{Processing Time}

The algorithm was tested using a Core2Duo processor running at $1.6 \mathrm{GHz}$ under the UNIX S.O. Preprocessing took less than six seconds for all data sets. Fine-tuning took ten sec./iteration. The number of iterations required to achieve a stable solution was between five and ten, resulting in a total processing time of less than two minutes.

\section{In vivo experiments}

Automatic placement of sat bands was reproducible across different scanning sessions on the same subject. Automatically placed sat bands were similar to the placement by a highly-trained human operator (Figure 6). Metabolite maps (Figure 7) of Cho, $\mathrm{Cr}$ and NAA in four of the eight encoded slices obtained with automatically placed bands of the suppression slices. The image quality is comparable with respect to uniformity and volume coverage and consistent with our previously published 2D results using manually-placed sat bands (19). Selected spectra from the same voxel in different slices demonstrate comparably low level of lipid contamination for manual and automatic placement of suppression slices (Figure 8); confirmed by their respective LC model fitted spectra (Figure 9).

\section{Discussion and Conclusions}

The performance of the fine adjustment was robust against variations of the initial suppression 
slice parameters from coarse adjustment, which is sufficiently fast, enabling online cross validation of the resulting parameters. One can compute the initial cost function and initial coverage percentages, and choose the best possible combination of the two. It was advantageous to decrease the initially computed distances $b_{i, l}$ of the inner planes to the center of coordinates by a factor $\beta, 0<\beta \leq 1$ in order to improve the lipid coverage. As expected, a decrease in the inner distance increased lipid coverage, while the initial value of outer volume coverage remained close to $100 \%$, which did not change significantly after the fine adjustment procedure. Varying the three cost function parameters did not result in significant differences in lipid coverage. In order to optimize non-lipid coverage, we choose $\beta=0.9, \mu=1.25^{-4}$ and eight iterations of the fine adjustment step to reach a stable solution.

Using a value of $\beta=0.9$, different values of $\varphi$ between $\pi / 6$ and $\pi / 2$ were tested. The largest solid angle produced the best results, whereas smaller angles led to insufficient lipid coverage due to irregularities in the surface. An interval between $\pi / 6$ and $\pi / 4$ in $\theta$ is suitable for the most brain data. Total coverage is reached slightly varying the thickness of the planes. Final results include $100 \%$ lipid coverage and less than $7 \%$ non-lipid coverage, independent of initialization.

The automated method presented here results in sat band settings similar to a highly-trained human operator. The method reduces operator interaction, it avoids variability due to human error in identifying lipid containing regions and optimization of sat band placement, and facilitates placement of sat bands for volumetric studies. Once fully integrated this method will save considerable setup time for short TE 3D-MRSI. To our knowledge, this is the first implementation of automated sat band placement on clinical scanners for brain MRSI, though a related method (11) to select an irregularly shaped volume inside brain using multiple sat bands with a block optimization algorithm has been presented. The method described in this paper enables flexible trade-off between lipid coverage and loss of peripheral brain regions due to saturation.

It is assumed that the peripheral lipid region to be covered forms a convex hull. This assumption is approximately true in the upper cerebrum. However, coverage of more inferior brain areas, such as cerebellum, requires additional suppression slices. The convexity assumption can be replaced by the more general assumption that any nonconvex closed surface can be fragmented into a finite set of convex surfaces. Then, further improvement includes adaptation to non-convex regions containing lipid.

Different anatomical areas inside the brain may have different importance in a given spectroscopic imaging application, or these areas may suffer from different degrees of magnetic field inhomogeneities. In order to address these issues, the cost function can be modified to have 
different weights for different areas of the brain. This reduces loss of brain tissue in critical regions at the expense of reduced brain coverage in other brain regions. Furhtermore, the method is not limited to sixteen bands, but for practical reasons, introducing more bands into the pulse sequences may not be feasible due to limitations in RF power deposition and loss in suppression efficiency due to $T_{1}$ relaxation as the duration of the train of suppression RF pulses increases. The sensitivity of spatial suppression to $B_{1}$ inhomogeneity and $T_{1}$ relaxation during the application of the suppression pulses can be mitigated using multiple repetitions of the suppression pulses with different flip angles as shown in (12). Alternatively, curved slice excitation enables more efficient coverage of lipid regions with reduced loss of lateral cortical brain regions. Recent developments in Transmit-SENSE technology reduce RF pulse widths making this approach more feasible. Although its implementation is technically challenging, the method described here is easily extended to bands constructed with any set of parametric functions with essentially the same computational cost.

Further improvement can be achieved by taking into account the transition width of the suppression slices and by compensating flip angle variation across the slices due to $B_{1}$ inhomogeneity using RF shimming (20). This will avoid the undesirable effects of partial lipid suppression in the transition regions and limit suppression of lateral brain tissue. In vivo experiments are currently limited to eight sat bands due to limitations in pulse sequence design that we are in the process of resolving. Future implementations in vivo will use up to sixteen sat bands.

\section{Acknowledgments}

Supported by NIDA 1 R01 DA14178-0, P41 RR08079 and the MIND Institute - Mental Illness and Neuroscience Discovery DOE Grant No. DE-FG02-99ER62764, S-0505/TIC/0223 (Madrid Government), TEC2008-02473 (Spanish MEC). We thank Dr. Stephen R. Dager (University of Seattle) and Dr. John van Meter (Georgetown University) for supporting in vivo tests of this methodology.

\section{References}

1. Atlas SW, Grossman RI, Hackney DB, Goldberg HI, Bilaniuk LT, Zimmerman RA. STIR MR imaging of the orbit. AJR Am J Roentgenol 1988;151(5):1025-1030.

2. Bydder GM, Young IR. MR imaging: clinical use of the inversion recovery sequence. J Comput Assist Tomogr 1985;9(4):659-675. 
3. Krinsky G, Rofsky NM, Weinreb JC. Nonspecificity of short inversion time inversion recovery (STIR) as a technique of fat suppression: pitfalls in image interpretation. AJR Am J Roentgenol 1996;166(3):523-526.

4. Shuman WP, Lambert DT, Patten RM, Baron RL, Tazioli PK. Improved fat suppression in STIR MR imaging: selecting inversion time through spectral display. Radiology 1991;178(3):885-887.

5. Spielman DM, Pauly JM, Macovski A, Glover GH, Enzmann DR. Lipid-suppressed single- and multisection proton spectroscopic imaging of the human brain. J Magn Reson Imaging 1992;2(3):253-262.

6. Posse S, Cuenod CA, Risinger R, Le Bihan D, Balaban RS. Anomalous transverse relaxation in $1 \mathrm{H}$ spectroscopy in human brain at 4 Tesla. Magn Reson Med 1995;33(2):246-252.

7. Mescher M, Tannus, A., Johnson, M. O., Garwood, M. Solvent Suppression Using Selective Echo Dephasing J Mag Reso, Series A 1996;123(2):226-229.

8. Star-Lack J, Nelson SJ, Kurhanewicz J, Huang LR, Vigneron DB. Improved water and lipid suppression for 3D PRESS CSI using RF band selective inversion with gradient dephasing (BASING). Magn Reson Med 1997;38(2):311-321.

9. Singh S, Rutt, B., Henkelman, R. . Projection presaturation: A fast and accurate technique for multidimensional spatial localization. J Magn Reson 1990;87:567-583.

10. Ryner L, Westmacott, G., Davison, N., Latta, P. Automated Positioning of Multiple Spatial Saturation Planes for Non-Cuboidal Voxel Prescription in MR Spectroscopy. ISMRM; 2005. p 350.

11. Venugopal N, McCurdy, B., Ryner, L. . Optimization of Outer Volume Suppression for Improved Prostate MR Spectroscopic Imaging. Medical Phisics 2006;33(6):2183.

12. Kiebel SJ, Friston KJ. Statistical parametric mapping for event-related potentials: I. Generic considerations. Neuroimage 2004;22(2):492-502.

13. Kiebel SJ, Friston KJ. Statistical parametric mapping for event-related potentials (II): a hierarchical temporal model. Neuroimage 2004;22(2):503-520.

14. Smith SM, Jenkinson M, Woolrich MW, Beckmann CF, Behrens TE, Johansen-Berg H, Bannister PR, De Luca M, Drobnjak I, Flitney DE, Niazy RK, Saunders J, Vickers J, Zhang Y, De Stefano N, Brady JM, Matthews PM. Advances in functional and structural MR image analysis and implementation as FSL. Neuroimage 2004;23 Suppl 1:S208-219.

15. Dougherty ER, Lotufo RA. Hands-on morphological image processing. Bellingham, Wash.: SPIE Optical Engineering Press; 2003. xvi, 272 p. p.

16. Sedgewick R. Algorithms in C. Reading, Mass.: Addison-Wesley; 1998. <v. 1-2 in 5 $>$ p.

17. Evans AC CD, Mills SR, Brown ED, Kelly RL, and Peters TM. 3D statistical neuroanatomical models from 305 MRI volumes. IEEE-Nuclear Sci Symp Med Imagine Conf 1993. p 1813-1817.

18. Posse S, Otazo R, Caprihan A, Bustillo J, Chen H, Henry PG, Marjanska M, Gasparovic C, Zuo C, Magnotta V, Mueller B, Mullins P, Renshaw P, Ugurbil K, Lim $\mathrm{KO}$, Alger JR. Proton echo-planar spectroscopic imaging of J-coupled resonances in human brain at 3 and 4 Tesla. Magn Reson Med 2007;58(2):236-244.

19. Provencher SW. Estimation of metabolite concentrations from localized in vivo proton NMR spectra. Magn Reson Med 1993;30(6):672-679.

20. Metzger GJ SC, Akgun C, Vaughan T, Ugurbil K, Van de Moortele. Local $\mathrm{B}_{1}^{+}$ shimming for prostate imaging with transceiver arrays at $7 \mathrm{~T}$ based on subject- 
dependent transmit phase measurements. Magnetic Resonance in Medicine 2008;59(2):396-409.

\section{Figure Legends}

Figure 1. Parameters of one of the bands.

Figure 2. Identification of the inferior edge of the MRSI volume. (a) at slice 120 (from the top), there is only one connected component (gray), which is assigned to the lipid volume, (b) at slice 128, several small connected components emerge. The largest region is marked as non-convex (white) (c) Some of the non-convex areas merge with the convex region at slice 136. All voxels below the non-convex area will be marked as well, as (d) shows.

Figure 3. Coarse adjustment of the bands.

Figure 4. Detailed evolution of the lipid and non-lipid (brain tissue and areas out of the head) covered for different values of $\beta$ and chosen values of $a, b, c$ and $\mu . \circ: \beta=0.8, \triangle: \beta=0.9$, $\nabla: \beta=1.0$.

Figure 5. Sagital, axial and coronal cuts of the obtained lipid mask, and complete outer view of the convex suppression area.

Figure 6. Automatically-placed sat bands have similar positions and orientations to those placed manually by a highly trained human operator for the same subject.

Figure 7. From automatically placed sat bands, metabolic maps of NAA, $\mathrm{Cr}+\mathrm{PCr}$, and total Cho of the four central slices obtained by 3D PEPSI acquisition (total 8 slices). The middle two show excellent image quality as compared to previous 2D maps (19).

Figure 8. Comparison of images from manual and automatic placements of sat bands show excellent agreement on the water suppressed images and the spectra for the same voxel in slice 4 .

Figure 9. Detailed spectra quantification from LC model fittings for the same voxel in Fig. 8 are in excellent agreement. 
